ISSN electrónico: 2602-8069

\title{
INVESTIGACIÓN Y DIFUSIÓN CIENTÍFICA: COMPETENCIAS ESENCIALES EN LA FORMACIÓN UNIVERSITARIA DEL PROFESIONAL EN CIENCIAS QUÍMICAS
}

Research and Scientific Dissemination:

Essential Competences in the University Training of the Professional in Chemical Sciences

Elithsine Elizabeth Espinel Armas Universidad Central del Ecuador eeespinel@uce.edu.ec

Mariana Gisell Calderón Defaz Universidad Central del Ecuador mgcalderon@uce.edu.ec 


\section{RESUMEN}

Los futuros profesionales que se educan en el campo de las ciencias químicas requieren de procesos académicos que apoyen el desarrollo de habilidades investigativas y refuercen las capacidades para la comunicación de resultados a la comunidad científica y a la sociedad. Así, la investigación científica constituye un eje fundamental en la formación del estudiante de la Facultad de Ciencias Químicas, y la integración de los ámbitos académico, de investigación y de vinculación con la sociedad es necesaria para alcanzar la misión institucional. El objetivo de este estudio fue identificar los medios que utilizan los estudiantes y docentes de la Facultad de Ciencias Químicas de la Universidad Central del Ecuador para comunicar los resultados de sus investigaciones.

El enfoque de esta investigación es cuantitativo y descriptivo. Se realizó el procesamiento de datos de los estudiantes graduados mediante un proyecto de investigación, por carrera y en la facultad, así como de los medios de difusión que se utilizaron para presentar los resultados, durante el período 2016-2018, a fin de establecer su relación. En ambos casos, los resultados se presentan en porcentajes y frecuencias absolutas. Además, la población de estudio perteneciente al sector docente aportó a esta investigación mediante entrevistas. Los docentes informaron sobre los medios de difusión utilizados, pósteres, ponencias y artículos científicos, a nivel nacional e internacional. Esto permitió concluir que el proceso de graduación en el período de estudio la difusión científica incrementó un $28 \%$.

Palabras Clave: Habilidades, investigación científica, producción científica, formación profesional, educación universitaria.

\section{ABSTRACT}

Future professionals who are trained in the field of chemical sciences require academic processes that support the development of research skills. Additionally, these must strengthen the abilities to communicate research findings to the scientific community and society. Scientific research constitutes a fundamental axis in the undergraduate training of students who belong to the Faculty of Chemical Sciences. On the other hand, the integration of the academic, research and community outreach areas is necessary to achieve the institutional mission. The aim of this study was to identify the strategies used by students and teachers from the Faculty of Chemical Sciences of Universidad Central del Ecuador to communicate their research findings. This is a quantitative and descriptive study. Data regarding graduate students per career and in the faculty for the period 2016-2018 were analyzed. Results are presented in percentages and absolute frequencies. In addition, professors contributed to this study through interviews. Professors mentioned that the main strategies used to communicate research findings were posters, conference presentations and scientific articles. In conclusion, the graduation processes that took place in the study period generated important scientific publications, over $28 \%$.

Keywords: Skills, scientific research, science communication, professional training, university education 


\section{Elithsine Elizabeth Espinel Armas - Mariana Gisell Calderón Defaz \\ INVESTIGACIÓN Y DIFUSIÓN CIENTÍFICA: COMPETENCIAS ESENCIALES \\ EN LA FORMACIÓN UNIVERSITARIA DEL PROFESIONAL EN CIENCIAS QUÍMICAS}

\section{INTRODUCCIÓN}

El desarrollo de habilidades en el campo de la investigación constituye una de las mayores fortalezas en la formación de los estudiantes de la Universidad Central del Ecuador (UCE) y de la Facultad de Ciencias Químicas (FCQ), particularmente. El componente investigativo ha sido un referente fundamental para la innovación en este centro de estudios, la cual, a través de su labor educativa integral ha encaminado esfuerzos para mejorar las condiciones de vida de su contexto. Por ello, los docentes en su praxis universitaria, descubren e indagan en aspectos concomitantes a las líneas de investigación y pertinentes a las necesidades en el área de la química y de la salud, según los lineamientos del Plan Nacional —Toda una Vida-. El profesor de la facultad, en el tratamiento de las ciencias fácticas, concentra su labor académica en el estudio de hechos, metodología y comprobación empírica, convirtiéndose en ejecutor de trabajos de investigación. Su misión es actuar como guía y mediador en el proceso de enseñanza-aprendizaje, lo cual permite que el estudiante desarrolle habilidades investigativas y divulgativas, parte de su formación integral como profesional.

Este estudio evidencia el diseño de proyectos y estudios de importante alcance, pero que, por diversas situaciones, no han sido difundidos o compartidos con la comunidad científica. Esta situación refleja una falta de concienciación sobre el impacto científico y el aporte que sus investigaciones pueden brindar a la sociedad.

Las Instituciones de Educación Superior (IES) deben promover todos los aspectos teóricos y prácticos para fomentar la educación integral de sus preprofesionales. La FCQ ha establecido programas de estudio que contribuyen al desarrollo y fortalecimiento de habilidades investigativas y divulgativas. La preparación académica ofrecida a los estudiantes garantizará la producción de las investigaciones científicas de gran calidad que podrán ser publicadas. Asimismo, la difusión científica es un proceso de relevante en las comunidades científicas, pues constituye un medio de fortalecimiento teórico-conceptual que permitirá responder a los problemas contextuales. Quienes se involucran en el campo científico de manera permanente deben fomentar una cultura de publicación y difusión de los avances científicos o tecnológicos y utilizar diversos medios para expresar sus mensajes. En consecuencia, las investigaciones tendrán contacto directo con el mundo científico y el mundo social, lo que propiciará la reflexión, análisis, e innovación, y conseguirá un fin aplicativo en la sociedad.

La difusión del conocimiento es una fase del trabajo científico que tiene lugar cuando la producción intelectual llega al usuario, es decir, va más allá del trabajo de edición; esto requiere hacer visible el trabajo editorial, significa ponerlo a la vista del usuario potencial a fin de facilitar su consulta. Sin visibilidad no hay uso del conocimiento que es en definitiva la razón de ser de su producción. Cada vez se presiona más a los investigadores sobre el impacto de sus publicaciones en la comunidad científica, medido por las citaciones; pero, además de esto, cada vez la comunidad científica en los países en desarrollo se ve más presionada para que dé respuesta a la necesidad de conocimiento sobre los problemas de la región, en la búsqueda de solución de estos. En otras palabras, el uso del conocimiento no sólo como enriquecedor de la ciencia, sino - como motor de desarrollo y como instrumento crítico en el proceso de construcción de sociedad" (Chaparro, F., 2001, p.22).

Desde este criterio, el proceso formativo en el ámbito universitario deberá garantizar el logro de resultados de aprendizaje que reflejen el desarrollo de habilidades de investigación en el estudiante y que contribuyan a superar el problema del abandono de muchas tesis de grado, las cuales son parte de un archivo de consulta, pero que nunca han sido difundidas entre la comunidad científica. Así lo dice Pedraza, citado por Gutiérrez \& Mayta (2010), 
54: "El paso por la universidad significa mucho más que el obtener un título profesional, sino contribuir al cumplimiento de la función de la misma: 'la producción de conocimiento nuevo"' (p. 54). Por ello, el futuro profesional tiene la misión de vincular la teoría con la práctica en el ejercicio laboral y responder a los requerimientos de su realidad social.

La difusión científica es el proceso de comunicar a la comunidad, de manera clara y fácil de comprender, los diferentes avances científicos y tecnológicos obtenidos en el área investigativa, utilizando diversos medios de difusión, artículos, pósteres o ponencias, para democratizar el conocimiento y aportar a los diferentes campos de la formación de un ser humano integral e innovador con un nivel cultural idóneo. Autores como Krauscopf \& Vera (1995) mencionan que "la insuficiencia de este, representa insuficiencia cultural, [ ] cuando en Latinoamérica, las revistas científicas han proliferado notablemente". A partir de esta consideración, se concluye que solo el enriquecimiento intelectual de una población contribuye, de forma directa e indirecta, con el desarrollo de la cultura y del sistema educativo, en especial, de la educación superior. La acción investigativa dirige la generación del conocimiento e innovación mediante la publicación de resultados en las revistas científicas nacionales e internacionales.

En Ecuador, las universidades no han escalado a niveles altos en relación con la difusión que les permita destacarse en el ámbito internacional. De manera particular, la UCE, aunque es reconocida por ser la primera institución pública de educación superior que ofreció una amplia gama tanto de facultades como de ofertas académicas y cumple con su misión mediante la investigación social y experimental, las labores de difusión científica todavía son escasas y carecen de periodicidad. Esta situación es contraproducente para el objetivo que persigue la Universidad y el esfuerzo realizado pierde valor. Este hecho refleja la falta de integración que debería existir entre las funciones sustantivas de la educación universitaria, pues es evidente la separación entre la academia, la investigación científica y la vinculación con la comunidad. Esto hace que persista el escaso impacto actual que tiene el conocimiento científico generado por investigadores nacionales en los ámbitos social y cultural del país y, a su vez, profundiza la invisibilización de la labor investigativa que realizan las universidades. Adicionalmente, la falta de inversión en la educación pública causa la ausencia de promoción de las revistas científicas.

Es necesario que el estudiantado cumpla con el requisito de titulación que consiste en la presentar un artículo científico estructurado que, posteriormente, será evaluado por el comité respectivo. Esta acción pone en la palestra a una generación que produce conocimiento con el fin de fortalecer el ámbito científico, tecnológico y humanístico de la sociedad.

Un importante avance en el ámbito educativo es que los estudiantes de pregrado y posgrado, producto de su formación académica, son capaces de identificar problemáticas, planificar y ejecutar investigaciones científicas y difundir los resultados obtenidos. La labor investigativa brinda varias ventajas a los estudiantes, tal como lo dice el autor Gutiérrez y Mayta (2003) no es una sino varias como "adquisición de experiencia, generación de criterio, formación del hábito y cultura de investigación, valoración personal, y una buena carta de presentación" (p.56). Por lo tanto, el proceso de investigación y publicación de artículos científicos en un medio de difusión, por ejemplo, una revista científica, fortalece el desarrollo de las capacidades individuales y colectivas, puesto que genera estudios orientados a resolver problemáticas de pequeña, mediana y gran escala.

Durante el proceso de enseñanza-aprendizaje, el docente enseña las pautas orientadoras al estudiante que le permitirán acercarse al contexto de la naturaleza misma de su área profesional. El trabajo investigativo fomenta en el alumnado una actitud científica y un conjunto de habilidades investigativas que serán útiles cuando se enfrente a un proceso de resolución de problemas en su campo profesional. García \& Ladino (2008) mencionan que 
[l]a enseñanza y el aprendizaje por medio de la investigación permite al docente proponer e implementar estrategias que conlleven a que el estudiante se aproximé [sic] al contexto en el que se desenvuelven los científicos, llevándolo a enfrentar situaciones que típicamente afrontan los científicos en su quehacer; por tanto, la estrategia de enseñanza y aprendizaje por medio de la investigación favorece el desarrollo de un tipo de competencias específicas que en este trabajo se denominaran competencias científicas.

La habilidad investigativa, en palabras de Machado (2008), define como: "El dominio de la acción que se despliega para solucionar tareas investigativas en el ámbito docente, laboral y propiamente investigativo con los recursos de la metodología de la ciencia" ( $p$. 350). Esta definición sugiere que estas habilidades se constituyen un eje transversal de los procesos sustantivos que reflejan destrezas lógico-formales, propias de la ciencia y la metodología investigativa. Estas se integran y se sintetizan en la habilidad de observación, recolección y organización de la información, uso de métodos de análisis de información, evaluación de métodos de investigación de acuerdo con el objeto de estudio, empleo de métodos de medición, de abordaje de problemas, manipulación de objetos y su registro, de interpretación y explicación, de predicción, interpretación de datos estadísticos, de experimentación, de discusión de resultados, argumentación y el uso del tiempo y el espacio.

Desde 2015 hasta la actualidad, la FCQ elabora el proceso de titulación para trabajos de investigación, como parte del Sistema de Calidad, basándose en las disposiciones generadas por la Dirección General Académica.

Fuentes de investigación: Si bien estas son diversas, es necesario destacar que los trabajos de titulación corresponde con las líneas de investigación de las carreras, proyectos de investigación de los docentes ganadores SENESCYT, Convenio ARES, proyectos Semilla y proyectos de investigación emblemáticos. Sin embargo, las últimas investigaciones de gran calidad fueron aquellas que surgieron de trabajos de titulación de pregrado y de los programas de maestría de la facultad. Se generaron artículos de revisión que describieron elementos claves que debían ser investigados. Estos artículos constituyeron la base de las propuestas innovadoras de investigación que luego se convirtieron en los trabajos de titulación de los estudiantes.

Dentro del análisis situacional de este estudio, se observa que en las carreras que conforman la FCQ de la UCE la tasa de graduación es baja. Además, el establecimiento de las Unidades de Titulación Especial, en 2015, contribuyó a que los egresados realicen exclusivamente Proyectos de Investigación, como mecanismo de graduación, lo cual permite la difusión de sus producciones científicas que se reflejaron en la presentación de artículos, pósteres y ponencias.

Bajo este antecedente, el estudio intentó responder la siguiente interrogante de investigación: ¿Cuál ha sido el nivel de difusión científica generado a partir de los trabajos de titulación de los estudiantes y profesores de la Facultad de Ciencias Químicas de la Universidad Central del Ecuador durante el período 2016-2018?

Los objetivos del estudio fueron los siguientes:

\section{Objetivo General}

Determinar el nivel de difusión científica alcanzado por estudiantes y profesores de las distintas carreras de la Facultad de Ciencias Químicas de la Universidad Central del Ecuador durante el período 2016-2018. 


\section{Objetivos Específicos}

- Establecer el número de estudiantes graduados por carrera y facultad, bajo la modalidad de proyecto de investigación, en el período 2016-2018, como potenciales comunicadores de resultados.

- Identificar las estrategias de difusión científicas predominantes de estudiantes y docentes producto de los trabajos de titulación desarrollados.

- Determinar la tasa de producción científica a partir de los proyectos de investigación realizados y el total de graduados bajo esta modalidad de titulación.

- Identificar las estrategias de difusión científica predominantes en la facultad.

La necesidad pedagógica e investigativa que orienta el estudio está considerada a partir el tratamiento de problemas o dilemas de la profesión como la escasa cultura divulgativa entre los estudiantes, práctica que no ha sido frecuente en su proceso formativo. Otro problema es la falta de una formación que integre la diversidad de disciplinas, la cual impulsaría la producción científica del alumnado. Cabe recalcar que la labor del profesional es aplicar sus conocimientos y herramientas investigativas para contribuir a la resolución de problemas que aquejan a la sociedad. Esta problemática ha sido tomada en consideración en el rediseño de las carreras de la facultad.

El aporte del estudio radica en recopilar datos cuantitativos sobre los estudiantes graduados de la FCQ, bajo la modalidad de proyecto de investigación, quienes deberían comunicar los resultados de sus investigaciones por medio de las estrategias de difusión, en distintos eventos o en las revistas de la universidad. También busca motivar a los estudiantes a apreciar a la investigación científica como fuente de desarrollo e innovación, durante la cual se promueve la excelencia y pertinencia educativa, para que se conviertan en actores dinámicos del proceso investigativo y divulgativo a nivel nacional e internacional.

\section{METODOLOGÍA}

El presente manuscrito corresponde a un artículo científico de investigación. El proceso metodológico empleado partió del análisis situacional y contextual del problema utilizando un árbol de problemas. Esto permitió plantear los objetivos y alcances de la investigación, para luego construir la ruta operativa para alcanzarlos. Asimismo, corresponde con un estudio cuantitativo y de nivel descriptivo, con apoyo de la investigación de campo sustentada con documentación bibliográfica y base de datos generada en el proceso de titulación.

En lo referente a la aprobación ética, se estructuró un protocolo de tratamiento de la información digital suministrada. Se estipuló que la información relacionada con los estudiantes sería revisada sin considerar sus nombres y apellidos, sino solo su registro de identidad. Además, se obtuvo el consentimiento informado de los docentes que participaron como directores o tutores de los trabajos de investigación. Ellos suministraron información sobre los mecanismos utilizados para la difusión de los resultados de las investigaciones.

La población que fue objeto de estudio estuvo conformada por los estudiantes graduados de la FCQ de la UCE durante los años 2016, 2017 hasta octubre de 2018. Esto corresponde a 234 individuos de las cuatro carreras que conforman la facultad. Estos datos se obtuvieron luego procesar la información contenida en la base de datos del proceso de graduación. El criterio de exclusión correspondió a los estudiantes que se graduaron bajo el mecanismo de titulación del Examen Complexivo, tomado por una sola ocasión en el 
proceso denominado Unidad de Titulación Especial. Fue necesario cuantificar el número estudiantes graduados por proyecto de investigación para establecer la relación entre medios de difusión científica y el total de graduados, tanto de la carrera como de la facultad. Adicionalmente, los docentes tutores que participaron en el estudio son profesores titulares de las carreras de la facultad, quienes describieron los mecanismos de difusión científica utilizados para comunicar los resultados de los trabajos de investigación elaborados, en forma conjunta, con los estudiantes.

Las variables consideradas para este estudio fueron las siguientes: trabajos de titulación y modalidades de difusión de hallazgos científicos.

Los instrumentos de investigación utilizados fueron una guía de observación y entrevistas a los directores de carrera, coordinadores de unidad de titulación y docentes tutores de la facultad. Las entrevistas permitieron establecer los trabajos de titulación que desembocaron en artículos científicos, pósteres, y/o ponencias. Los datos recopilados durante el estudio fueron analizados utilizando estadística descriptiva, para determinar las frecuencias absolutas y los respectivos porcentajes. Finalmente, se estableció la tasa de producción científica generada en la facultad.

\section{RESULTADOS}

Los resultados se configuran desde dos escenarios: estudiantes por carrera que se graduaron en el período 2016 (1), 2017 (2) y 2018 (3), este último con datos hasta octubre, bajo la modalidad de proyectos de investigación, y sobre los medios de difusión utilizados por carreras en este período.

\section{Graduados por proyecto de investigación}

Resulta importante identificar el número de graduados bajo la modalidad de trabajo de investigación, especialmente, por carrera y por facultad, para distinguirlos de las otras que se ofrecen para obtener el título de grado.

Gráfico 1. Estudiantes graduados con proyecto de investigación Química Farmacéutica

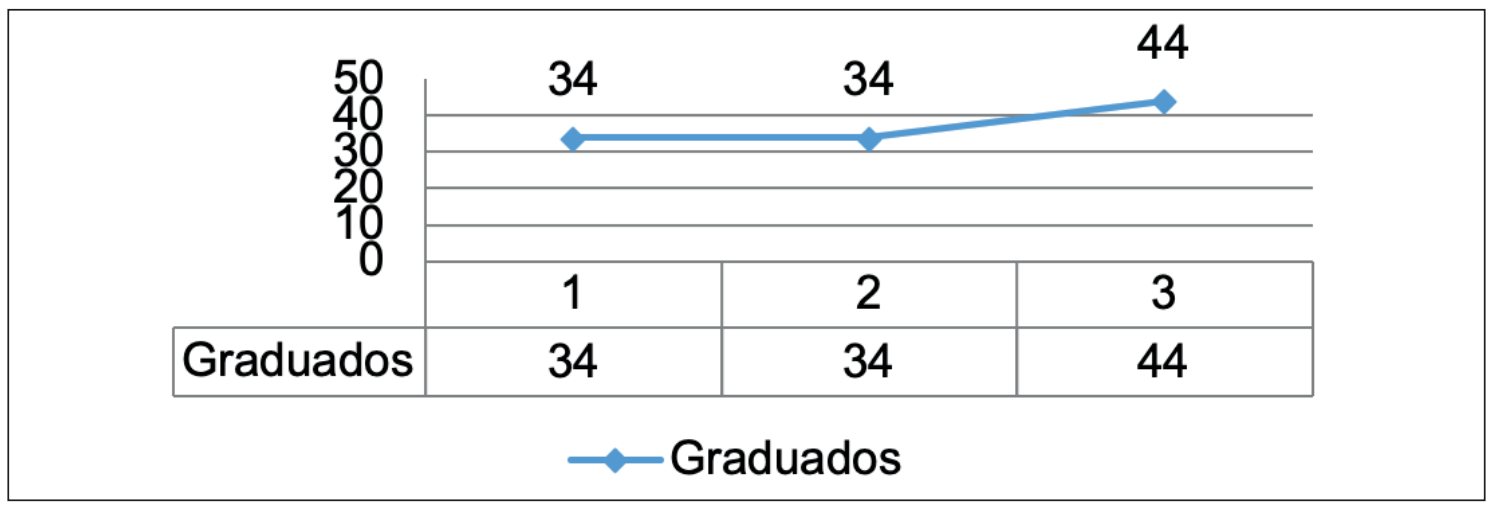

Fuente: Elaboración propia de las autoras (2018). Base de datos Secretaría de Subdecanato FCQ 
En la carrera de Química Farmacéutica (QF), según en el gráfico 1, se graduaron 112 profesionales en la modalidad proyecto de investigación. Se evidencia el crecimiento del número de graduados en el tercer año, objeto de análisis, en un $29.4 \%$, producto de la organización de la Unidad de Titulación Especial, la cual estandariza los procesos y cumplimiento de plazos.

Gráfico 2. Estudiantes graduados con proyecto de Investigación en Bioquímica Clínica

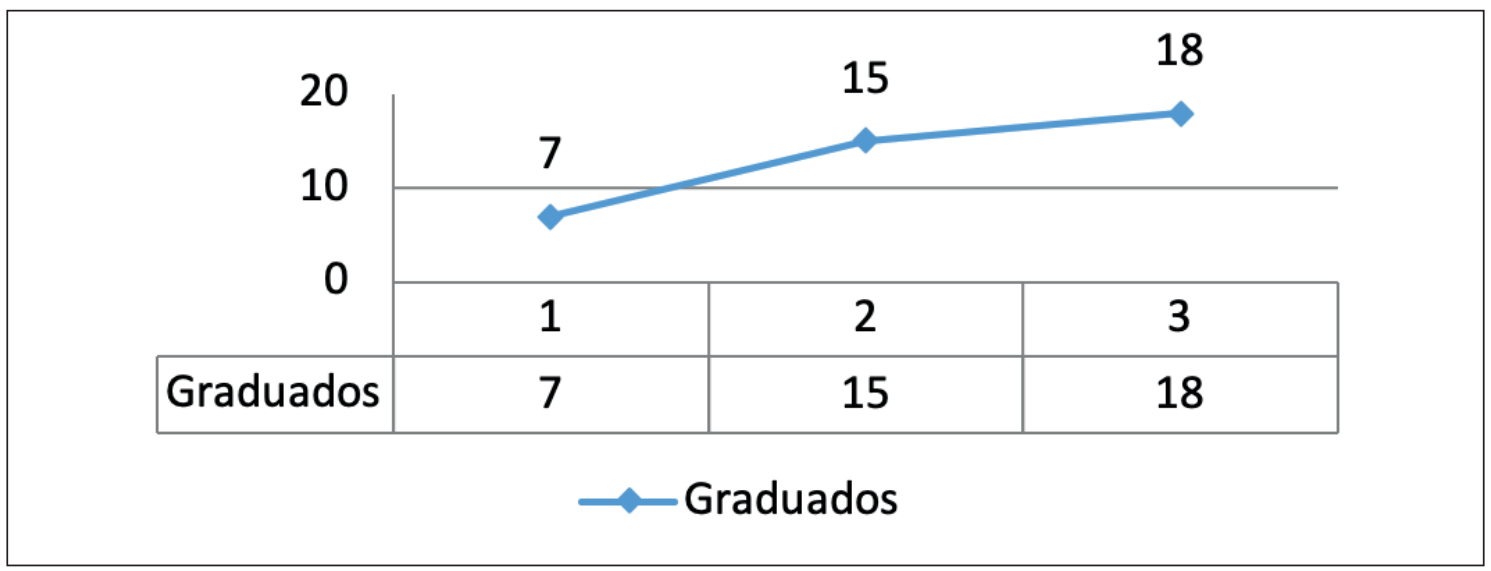

Fuente: Elaboración propia de las autoras (2018). Base de datos de Subdecanato FCQ

En el gráfico 2, sobre los estudiantes graduados con proyecto de investigación de la carrera de Bioquímica Clínica (BC), se muestra un crecimiento sostenido, de un $114 \%$, y 20 $\%$ en el período analizado.

Gráfico 3. Estudiantes graduados con proyecto de investigación en Química de Alimentos

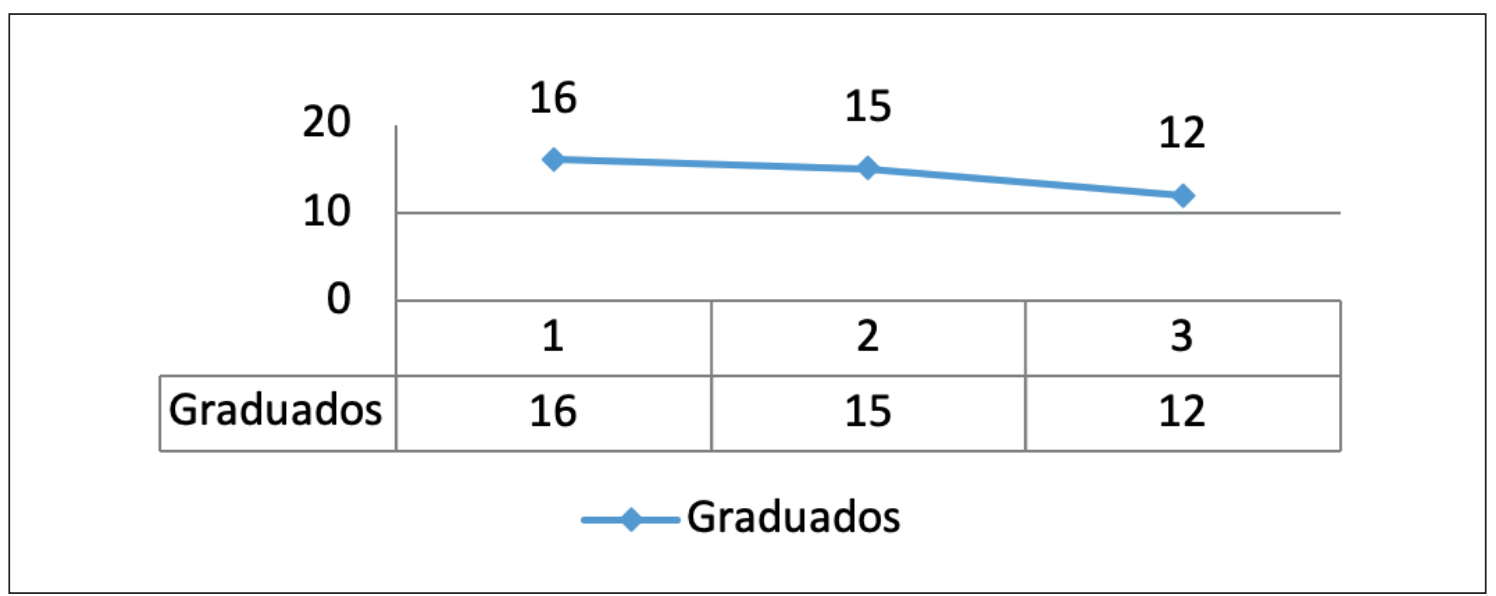

Fuente: Elaboración propia de las autoras (2018). Base de datos Secretaría de Subdecanato FCQ 
En el gráfico 3, se establece el número de estudiantes graduados con proyecto de investigación de la carrera de Química de Alimentos (QA). Se observa un decrecimiento del $6 \%$ en relación con el 2016, y del 20 \% respecto al año 2017.

Gráfico 4. Estudiantes graduados con proyecto de investigación Química

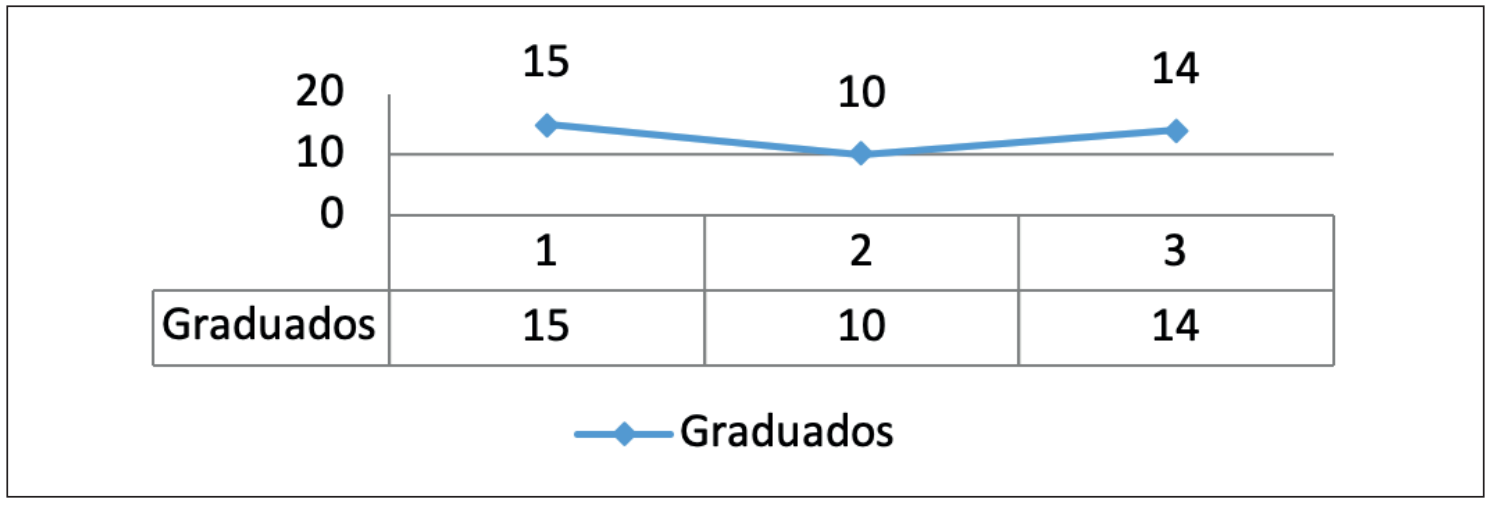

Fuente: Elaboración propia de las autoras (2018). Base de datos Secretaría de Subdecanato FCQ

En el gráfico 4, sobre los graduados con proyecto de investigación de la carrera de Química (Q), se evidencia un decrecimiento del $33 \%$ en relación con el 2016 , mientras que el 2018 se incrementa en un $40 \%$ respecto del año 2017.

En un análisis integrado anual de las carreras se presentan los siguientes resultados por cada año:

Gráfico 5. Estudiantes graduados por proyecto de investigación FCQ 2016

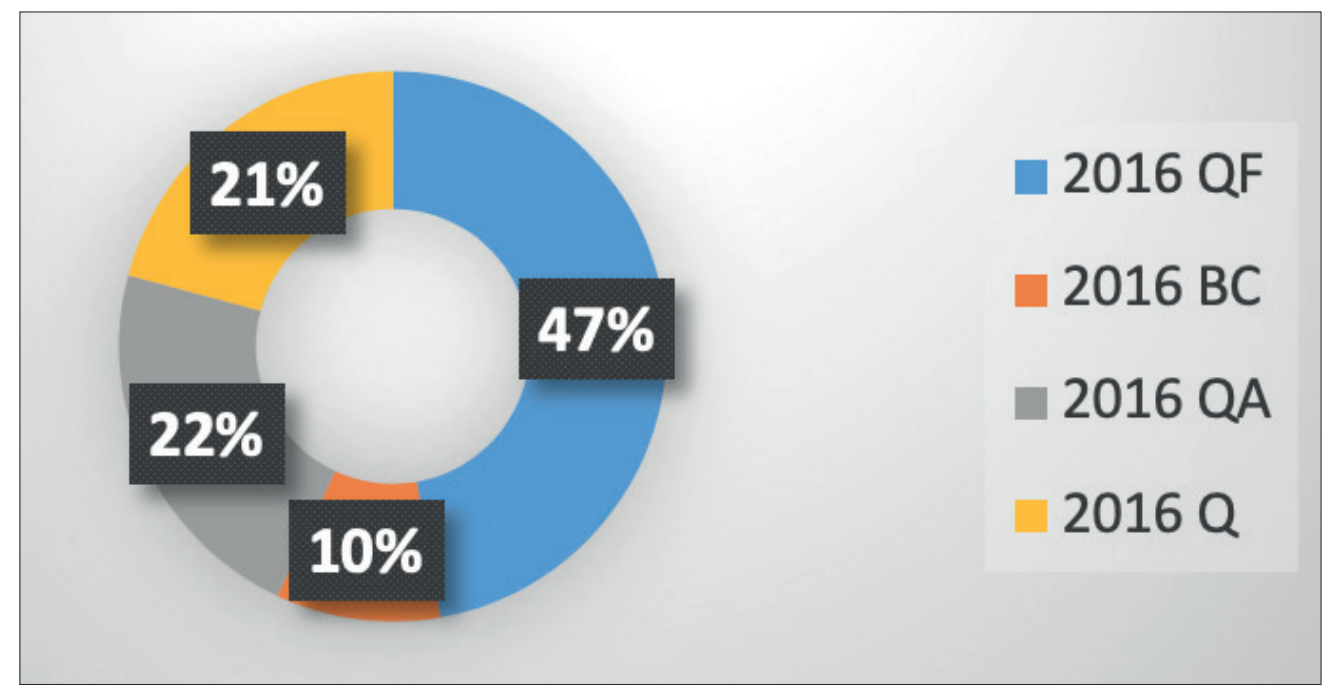

Fuente: Elaboración propia de las autoras (2018). Base de datos Secretaría de Subdecanato FCQ

En el gráfico 5, en el 2016, los estudiantes de la FCQ conforman de 72 graduados, de los cuales $47 \%$ son de QF, $10 \%$ de BC, $22 \%$ de QA y $21 \%$ de $Q$. 


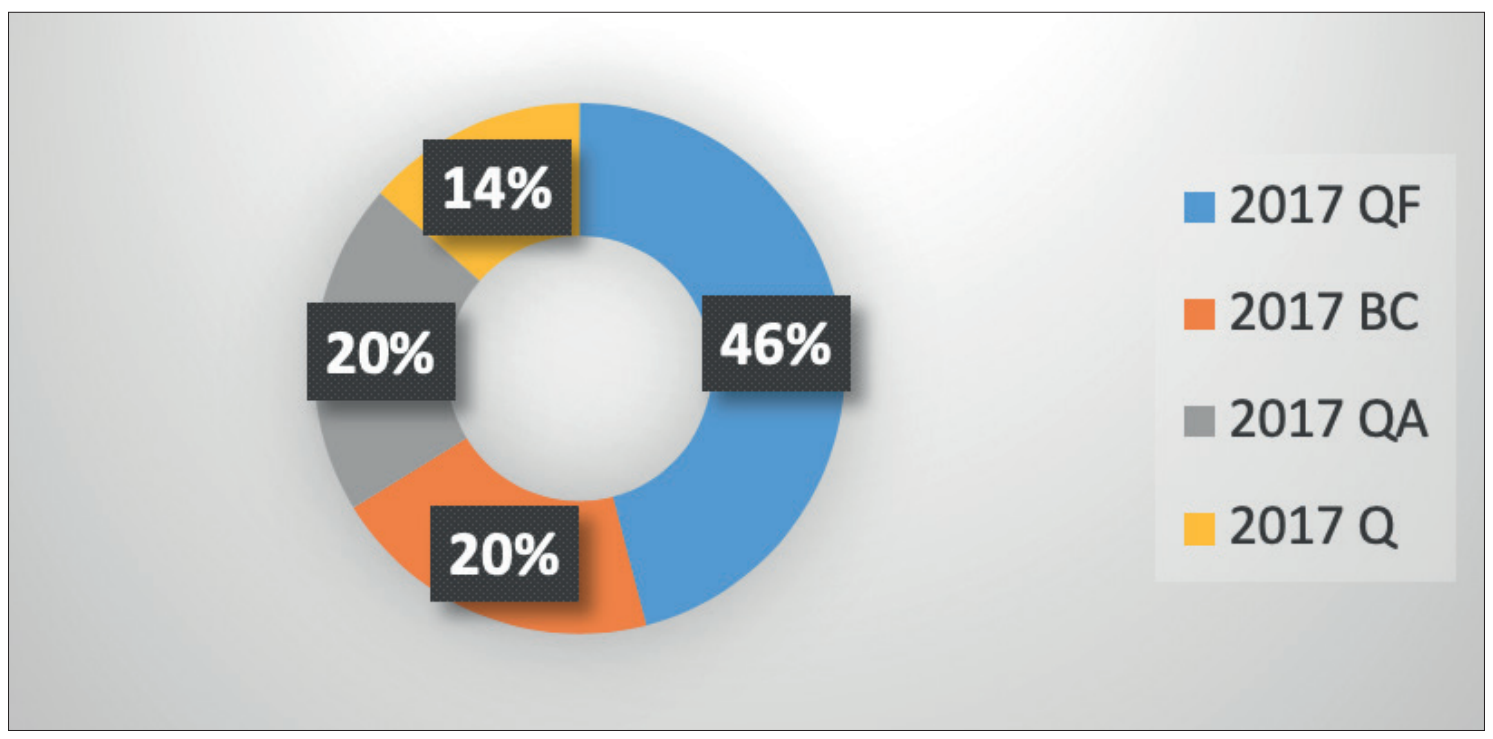

Fuente: Elaboración propia de las autoras (2018). Base de datos Secretaría de Subdecanato FCQ

El gráfico 6, en el 2017, el número de graduados corresponde a 74 estudiantes y comprende $46 \%$ a QF, $20 \%$ de BC, $20 \%$ QA y $14 \%$ de Q.

Gráfico 7. Estudiantes graduados por proyecto de investigación FCQ 2018

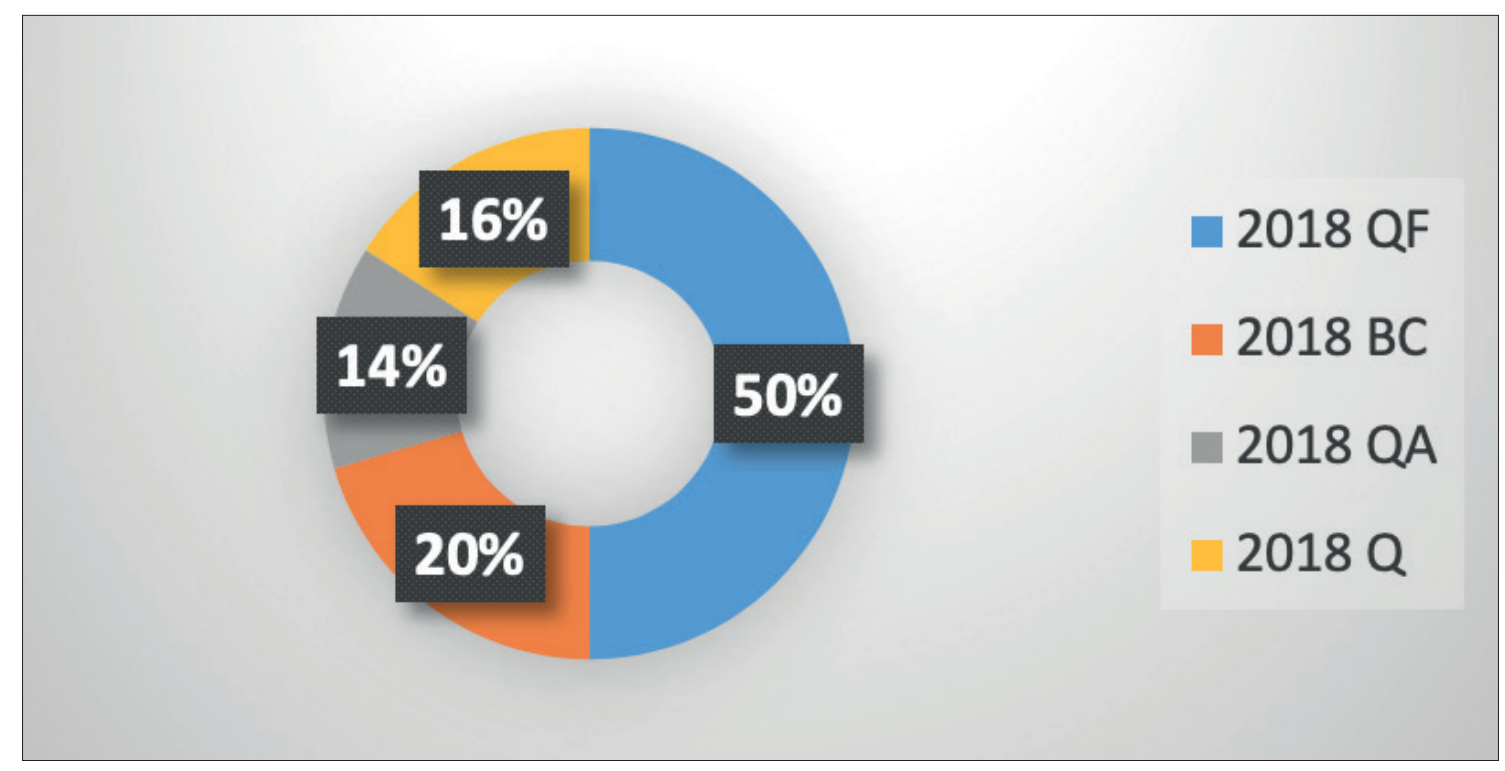

Fuente: Elaboración propia de las autoras (2018). Base de datos Secretaría de Subdecanato FCQ

En el gráfico 7, en el 2018, el número de graduados por proyecto de investigación, corresponde a 88 estudiantes. El $50 \%$ corresponden a QF, el $20 \%$ de BC, el $14 \%$ de QA y $16 \%$ de $Q$. 
Gráfico 8. Estudiantes graduados por proyecto de investigación FCQ 2016- 2018

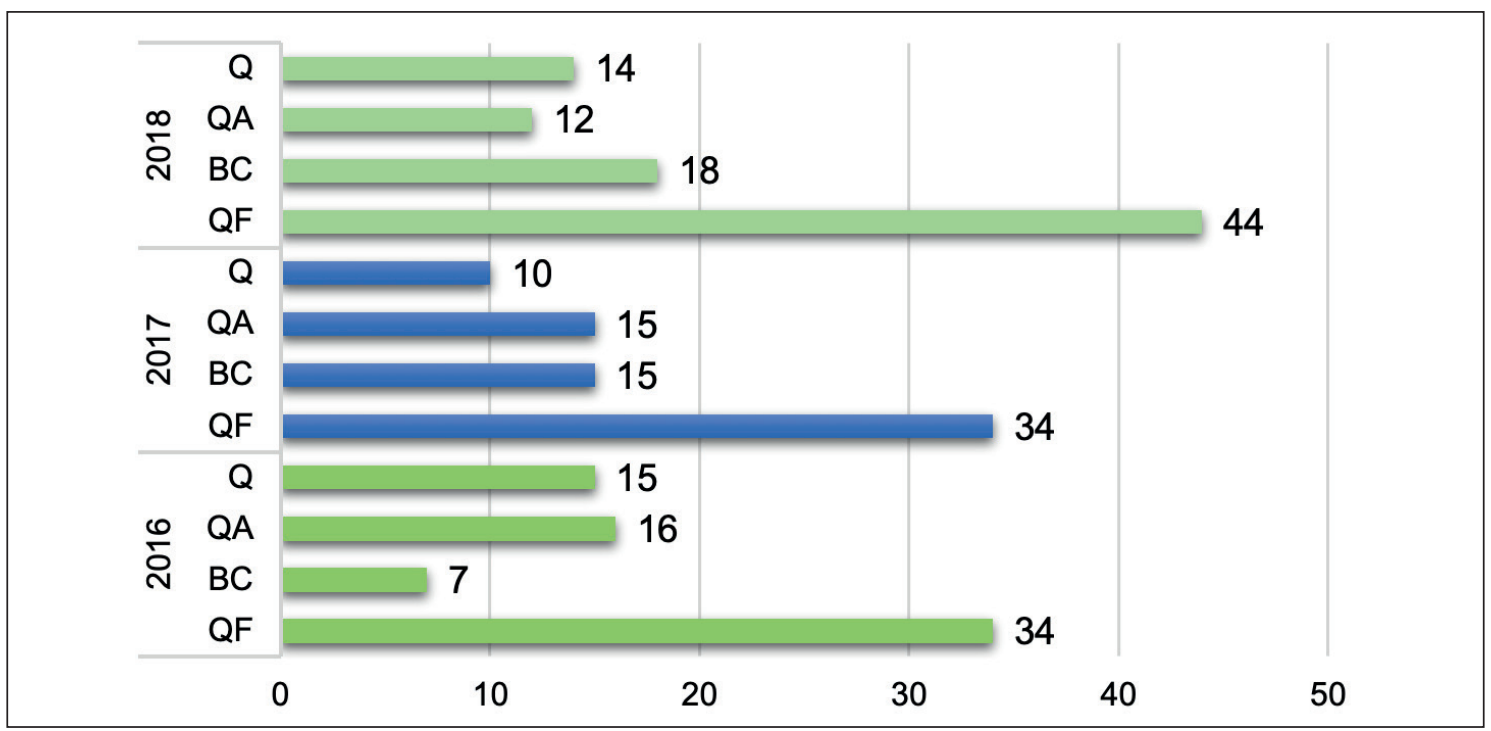

Fuente: Elaboración propia de las autoras (2018). Base de datos Secretaría de Subdecanato FCQ

Los resultados obtenidos, en el gráfico 8 , muestran el número de graduados de las cuatro carreras que corresponden a la FCQ, los cuales se han incorporado como profesionales en el período 2016-2018: 112 químicos farmacéuticos, 40 bioquímicos clínicos, 43 químicos de alimentos y 39 químicos, bajo la modalidad de proyecto de investigación, en total: 234 profesionales.

Medios de difusión científica

Los datos sobre los medios utilizados para la difusión científica, según información proporcionada por docentes tutores durante el período referido en el estudio, fueron los siguientes:

Gráfico 9. Medios de difusión científica utilizados por los graduados de Ciencias Químicas 2016-2018

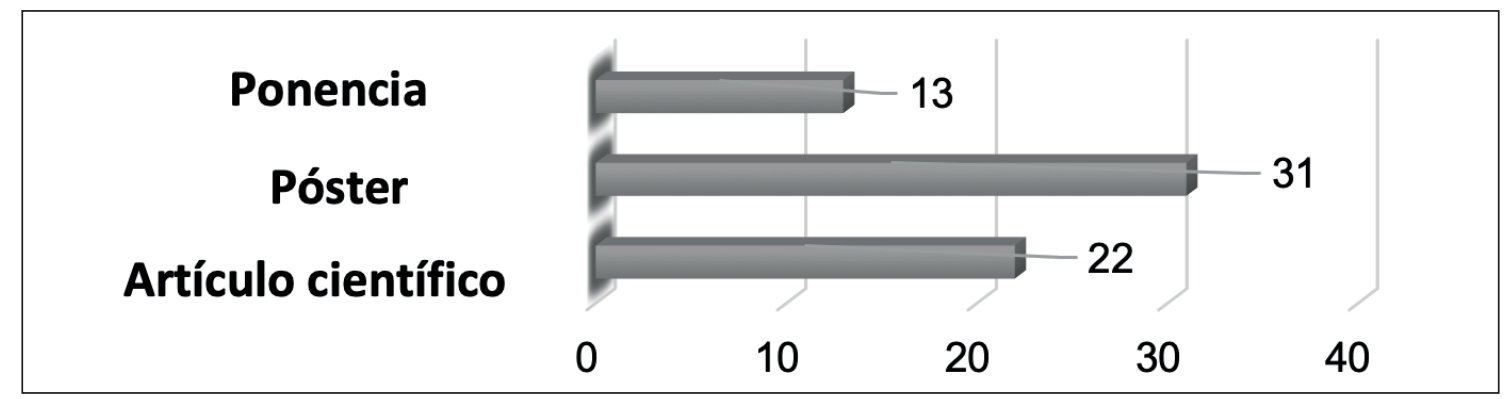

Fuente: Elaboración propia de los autores (2018). Registro de docentes. FCQ 


\section{Elithsine Elizabeth Espinel Armas - Mariana Gisell Calderón Defaz \\ INVESTIGACIÓN Y DIFUSIÓN CIENTÍFICA: COMPETENCIAS ESENCIALES \\ EN LA FORMACIÓN UNIVERSITARIA DEL PROFESIONAL EN CIENCIAS QUÍMICAS}

Los datos recopilados y mostrados en el gráfico 9 indican que se han realizado 66 publicaciones científicas, de las cuales mayoritariamente corresponden a pósteres (47\%), seguidos de artículos (33\%) y, finalmente, ponencias (20\%), lo cual evidencia el aporte al conocimiento que se realiza en las aulas de la facultad.

\section{Tasa de difusión cientifica en la facultad}

Hay que resaltar que las 66 publicaciones científicas de los estudiantes y docente tutores de proyectos de investigación corresponden al $28.20 \%$ en relación con el total de graduados por trabajo de investigación, en la facultad, durante el período 2016-2018.

\section{Estrategias de difusión predominante en la facultad}

Las estrategias de difusión utilizadas fueron pósteres, artículos científicos y ponencias en congresos, y su alcance es a nivel nacional e internacional. De estos se evidencia predominio de la participación, con pósteres, en congresos o seminarios vinculados al área del conocimiento de la facultad.

\section{DISCUSIÓN}

Los graduados que conforman la población de análisis corresponden a las cuatro carreras que oferta la facultad, y, en el período 2016-2018, recibieron su título profesional en un total de 234, quienes se graduaron bajo la modalidad proyecto de investigación.

En el artículo 21 del Reglamento de Régimen Académico se determinan las unidades de organización curricular y, entre ellas, la Unidad de titulación, que por medio de éste proceso se da viabilidad a las innovaciones y propuestas de investigación (CES, 2018). El 2 de junio de 2015, la Comisión Académica Permanente del Honorable Consejo Universitario aprobó el Instructivo de la Unidad de Titulación Especial de la Universidad Central del Ecuador, remitido por la Dirección General Académica, la Guía Operativa de la Unidad de titulación Especial del 5 de octubre de 2015, el 31 de marzo se aprueba y publica un último Instructivo General de Titulación de Grado en marzo de 2018.

Este proceso busca viabilizar la eficacia y efectividad de los resultados de aprendizaje mediante una acción investigativa más cercana a la realidad circundante, desarrollo de trabajos de experimentación, actividades autodirigidas, entre otras, que se consolidan con la planificación, seguimiento y acompañamiento, cronogramas de ejecución, cumplimiento de condiciones éticas y la difusión de resultados.

Las modalidades de titulación fijadas en la Unidad de Titulación Especial 2015 y aprobadas por los organismos pertinentes son Examen Complexivo, Proyecto de Investigación, Artículos de Revisión y Proyectos Integradores (Universidad Central del Ecuador, 2018). En vista de la naturaleza de las carreras de Ciencias Químicas, durante todo su período formativo los procesos de observación y experimentación sistemáticas son desarrollados en la mayoría de las asignaturas, lo que permite alcanzar el perfeccionamiento y afianzamiento de las habilidades investigativas. En consecuencia, los estudiantes toman la decisión exclusiva sobre el desarrollo de trabajo de investigación para su titulación.

Adicionalmente, desde sus inicios en la facultad, la modalidad de investigación denominada tesis era la única manera que los estudiantes de las carreras disponían para graduarse. Esta preferencia se muestra también la facultad de química de la UNAM, que 


\section{Elithsine Elizabeth Espinel Armas - Mariana Gisell Calderón Defaz \\ INVESTIGACIÓN Y DIFUSIÓN CIENTÍFICA: COMPETENCIAS ESENCIALES \\ EN LA FORMACIÓN UNIVERSITARIA DEL PROFESIONAL EN CIENCIAS QUÍMICAS}

señala lo siguiente: "entre enero de 2007 y octubre de 2009 se titularon 1471 egresados, siendo la tesis experimental y su correspondiente defensa ante un jurado de tres sinodales, la opción de titulación de mayor preferencia entre los estudiantes de nuestras cinco carreras (Facultad de Ciencias Químicas. Universidad Autónoma de México, 2017). Es decir, el 78.2 $\%$ de investigadores prefieren esta alternativa que concluye con artículo científico arbitrado.

Los resultados sobre el número de graduados con trabajo de investigación evidencia además la respuesta que da la Facultad de Ciencias Químicas de la Universidad Central del Ecuador a la sociedad, que se basa en la necesidad latente de los empleadores que, de manera permanente, requieren de profesionales químicos formados en habilidades investigativas, para desempeñarse en el ámbito laboral o esferas de actuación correspondientes y generar propuestas en beneficio de los consumidores o beneficiarios. De esta manera la formación se articula con el perfil de egreso.

Identificar las necesidades priorizadas en los planes de desarrollo nacional, provincial, local, regional, que les permita resolver los problemas de la sociedad en relación al Plan Nacional de Desarrollo, Plan de desarrollo Provincial y Metropolitano, Plan Nacional de Ciencia y Tecnología, la Innovación y los Saberes Ancestrales y la Matriz Productiva, para promover el desarrollo del sector productivo de los medicamentos y alimentos y mejoramiento de la calidad de vida de la población a través de la provisión de productos de uso y consumo humano de calidad e inocuos y la oferta de servicios farmacéuticos y de salud de calidad (Facultad de Ciencias Químicas, 2016).

La información referida por el INEC, en el 2010, refuerza lo anterior, pues muestra que el $0.3 \%$ del total de trabajadores en el Ecuador se encontraban en el sector farmacéutico y que a la vez se relaciona con el crecimiento de la industria farmacéutica, para aquel entonces del $15 \%$ (Jarrín, 2013). "La tasa de empleo en el sector de servicios sociales y de salud en el Ecuador en el 2018 alcanzó el 13.6\%" (INEC, 2018). Otra referencia es la en la página web de la Asociación de Laboratorios Farmacéuticos Ecuatorianos donde dice que "el 96\% de los trabajadores del sector farmacéutico a nivel mundial gozan de estabilidad laboral, en la mayoría de los casos cuentan con contrato indefinido y sus empleadores son empresas responsables y reconocidas" (Farma, 2017). Estos indicadores destacan un escenario importante para acceder a las plazas de trabajo en el sector referido.

Durante estos tres años y gracias al énfasis que se ha otorgado a la investigación como una disciplina científica que problematiza la realidad, y un instrumento que vincula a los planes nacionales con los requerimientos sociales, esta se constituye en el eje transversal de todo proceso de aprendizaje y de generación de conocimiento según las necesidades del contexto. Esto permite promover en los futuros profesionales un deseo por vincular la teoría con la práctica, describir la realidad, entender sus componentes desde el ejercicio causa y efecto, dar solución a los problemas y socializar resultados bajo estándares con rigor científico. Asimismo, las estrategias comunicativas también son diversas, por lo tanto, la difusión científica con participación directa y activa de los estudiantes ha crecido por medio de la presentación de ponencias, pósteres y artículos científicos presentados a nivel nacional e internacional.

La investigación es un proceso que recién culmina con la publicación del manuscrito; el ser estudiante o graduado no determina la calidad de una investigación, sino mas bien la rigurosidad científica con la que se haya realizado, por lo cual es indispensable cambiar el mal hábito que tienen los estudiantes de medicina de Latinoamérica de no publicar sus investigaciones. Para ello es necesario mejorar la calidad de sus trabajos, difundir la importancia, donde y como [sic] publicar, generar espacios de capacitación en publicación, brindar incentivos que motiven la generación de una cultura de publicación en Latinoamérica. (Gutiérrez \& Mayta, 2003, p. 59). 


\section{Elithsine Elizabeth Espinel Armas - Mariana Gisell Calderón Defaz \\ INVESTIGACIÓN Y DIFUSIÓN CIENTÍFICA: COMPETENCIAS ESENCIALES \\ EN LA FORMACIÓN UNIVERSITARIA DEL PROFESIONAL EN CIENCIAS QUÍMICAS}

Este planteamiento no es distante de lo que sucede en Ecuador y en el sistema universitario. Los procesos investigativos en la mayoría de los casos se realizan con el único propósito de culminar la carrera y como requisito de graduación. Por ello, es importante destacar que

[s]e podría, entonces asegurar que la investigación científica y la publicación del artículo científico son procesos interrelacionados; es común estimar que la investigación concluye cuando se obtienen los resultados, mediante la entrega del informe o con la respectiva disertación, sin embargo, es importante tender hacia la cultura de la divulgación de los trabajos realizados, mediante la escritura, esto afirmará la calidad de la investigación realizada, como lo sostiene (Mari, J., s.f.): "la investigación científica formal y seria termina con la publicación del artículo científico; sólo así tu contribución pasará a formar parte del conocimiento científico". (Hidalgo y Espinel, 2017, p. 44).

Lo señalado se respalda con el siguiente pronunciamiento:

"[...] es importante tener en cuenta que al término de un proceso investigativo o de producción científica, cultural o académica hay un deber: dar a conocer lo que se ha descubierto o comprendido (previa evaluación), con el fin de propiciar el diálogo entre el conocimiento y la sociedad, cada vez más alfabetizada, técnica e instruida; hecho que sin lugar a dudas exige un adecuado manejo de la escritura y sus diferentes convenciones para acometer con éxito innumerables tareas [...]" (Sánchez, A., 2011, p. 20)

El marco estatutario universitario, mediante Dirección de Comunicación y Cultura, como una de sus funciones, respalda la producción científica, como dice a continuación:

"[...] Promover la publicación de todo tipo de material impreso y digital para divulgar la producción del conocimiento, el arte y la cultura [...] Difundir los programas académicos, de investigación y vinculación con la sociedad mediante la actividad comunicacional" (Universidad Central del Ecuador, 2016, p. 26)

Además, se hace referencia a las funciones inherentes a la temática a la Dirección de Investigación en el registro de publicaciones científicas, ejecución de sistema de calificación de publicaciones para mantener revistas científicas institucionales, generación de normativa sobre propiedad intelectual (Universidad Central del Ecuador, 2016). De tal forma, existe el sustento para sustentar la difusión de resultados de investigaciones. Aparte, este es un indicador de evaluación relevante y es necesario reforzarlo a nivel universitario.

Los medios de difusión de mayor que más utilizan estudiantes y docentes, según los resultados obtenidos, en ese orden, son el póster, el artículo científico y las ponencias. Estos resultados difieren de los presentados por Morocho (2018) sobre la producción científica en Ecuador, en el período 2010-2016, donde señala la preferencia en un $78.7 \%$, equivalentes a más de 4000 , por los artículos científicos; las conferencias en un segundo lugar, con 1011 documentos y, finalmente, los libros y capítulos en un $1.53 \%$, con 78 documentos.

\section{CONCLUSIONES}

Las conclusiones que se extraen son las siguientes:

Los informes de investigación presentados por los graduados constituyen un medio de difusión, pues son recopilados en el repositorio digital universitario. Sin embargo, un 28 $\%$ se convierte en otras formas de difusión. Aunque existe un marco normativo que estimula los procesos de investigación y divulgación, estos deben ser aún optimizados, para alcanzar 


\section{Elithsine Elizabeth Espinel Armas - Mariana Gisell Calderón Defaz \\ INVESTIGACIÓN Y DIFUSIÓN CIENTÍFICA: COMPETENCIAS ESENCIALES \\ EN LA FORMACIÓN UNIVERSITARIA DEL PROFESIONAL EN CIENCIAS QUÍMICAS}

resultados más eficientes y efectivos de los estudios investigativos que se producen en las universidades y centros de investigación.

Los estudiantes de la FCQ denotan un gran potencial investigativo, sin embargo, las producciones científicas de sus trabajos aún es limitada, pues generalmente ocurre que una vez terminada la investigación no demuestran interés de difundir los resultados.

El mecanismo de titulación denominado Proyecto de Investigación refleja las habilidades investigativas desarrolladas en el proceso de aprendizaje, pero relega la elección de otras modalidades que se ofrecen en las carreras como el examen complexivo, los proyectos integradores y los artículos de revisión.

El modelo de interacción entre estudiantes de posgrado, pregrado, docentes e investigadores, en un esquema multinivel, multidisciplinario e interinstitucional, enmarcado en una línea de investigación clara y definida, permite una generación eficiente del conocimiento y de sus aplicaciones y contribuye a construir una cultura de investigación en la universidad.

Es recomendable conformar bases de datos de los trabajos científicos difundidos para determinar el impacto y la proyección científica de las distintas unidades académicas.

Los planes y programas de estudio de las carreras de la facultad deben fortalecer la escritura científica para revistas y procesos de publicación, de manera que sea una práctica cotidiana, y no ocasional, para evitar inseguridad e imprecisión.

Es necesario motivar al estudiantado hacia el propósito de la ciencia que es comunicar los hallazgos científicos, más aún de los procesos experimentales que demandan habilidades cultivadas en el aula.

\section{BIBLIOGRAFÍA}

Baptista, P., \& Collado, C. \&. (2014). Metodología de la investigación. México: McGrawHill.

CES. (2019). República del Ecuador. Reglamento De Régimen Académico. http://doi.org/DOI 10.1111/ j.1438-8677.2009.00206.

Chaparro, F. (Abril de 2001). Conocimiento, aprendizaje y capital social como motor de desarrollo.

Facultad de Ciencias Químicas. (2016). Pertinencia y plan curricular.

Facultad de Ciencias Químicas. Universidad Autónoma de México. (2017). Tesis experimental, la opción de titulación predominante en FQ. Retrieved from https://quimica.unam.mx/tesis-experimental-la-opcion-de-titulacion-predominante-en-fq/

Farma, C. (2017). Sector farmacéutico impulsa la tasa de empleo mundial. Retrieved from http://farma. ebizor.com/sector-farmaceutico-impulsa-empleo/

García, G., \& Ladino, Y (2008). Desarrollo de competencias científicas a través de una estrategia de enseñanza y aprendizaje por investigación.

Gutiérrez, C., \& Mayta, P. (2003). Publicaciones desde el Pre Grado en Latinoamérica: Importancia, limitaciones y alternativas de solución. Obtenido de UAEM: Redalyc.org: http://www.redalyc. org/html/717/7

Hidalgo, D. y Espinel, E. (2016). Divulgación Académica y Científica. Quito: Servicios Publicitarios.

INEC. (2018). www.ecuadorencifras.gob.ec/. Obtenido de www.ecuadorencifras.gob.ec/.

Jarrín, P. (2013). El sector farmacéutico mundial y ecuatoriano. Un análisis de las implicaciones del otorgamiento de licencias obligatorias de patentes. Repositorio.puce.edu.ec/bitstream/ handle/22000/6109/T-PUCE-6346.pdf?...1...y.Jarrín, P. J. (Octubre de 2013). repositorio. puce.edu.ec/bitstream/handle/22000/6109/T-PUCE-6346.pdf?...1...y. Obtenido de repositorio.puce.edu.ec/bitstream/handle/22000/6109/T-PUCE-6346.pdf?...1...y.

Krauskopf, M., \& Vera, M. (Julio de 1995). Indicadores y estrategias para la consolidación de revistas científicas. Obtenido de Las revistas latinoamericanas de corriente principal: http://www. interciencia.org.ve 
Martínez D.; \& Márquez D., Las habilidades investigativas como eje transversal de la formación para la investigación. Cuba. 2014

Mari, J. (s.f.). Manual de Redacción Científica*. Puerto Rico: Universidad de Puerto Rico.

Morocho, J. (2018). Análisis Bibliométrico de la Producción Científica en el Sistema de Educación Superior Ecuatoriano, 146.

Sánchez, A. (2011). Manual de redacción académica e investigativa: cómo escribir, evaluar y publicar artículos. Medellín: Católica del Norte Fundación Universitaria.

UCE (2016). UCE en cifras. Obtenido de http://reportes.uce.edu.ec/Matriculados/Matriculados.aspx Universidad Central del Ecuador. (2016). Estatuto Universitario. Quito: Editorial Universitaria.

Universidad Central del Ecuador. (2018). Facultades y Modalidades de titulación. Retrieved from http://www.uce.edu.ec/ 